\title{
High-Pressure Phase Transitions and Thermodynamic Behaviors of Cadmium Sulfide
}

\author{
J.J. TAN ${ }^{a, b, *}, Y . \mathrm{LI}^{a}$ AND G.F. JI ${ }^{b}$ \\ ${ }^{a}$ SSchool of Science, East China Institute of Technology, Nanchang, 330013, China \\ ${ }^{b}$ Laboratory for Shock Wave and Detonation Physics Research, Institute of Fluid Physics \\ Chinese Academy of Engineering Physics, Mianyang, 621900, China \\ (Received August 2, 2010; revised version April 5, 2011; in final form May 10, 2011)
}

\begin{abstract}
The pressure-induced phase transitions of cadmium sulfide semiconductor in both zinc-blende and wurtzite structures are investigated by ab initio plane-wave pseudopotential density functional theory with the local density approximation. On the basis of the fourth-order Birch-Murnaghan equation of state, the phase transition pressures $P_{\mathrm{t}}$ are determined by the enthalpy criterion. It is found that the phase transitions occur at pressure of $2.57 \mathrm{GPa}$ (zinc blende-rocksalt structure) and $2.60 \mathrm{GPa}$ (wurtzite-rocksalt structure), respectively. The equilibrium structural parameters, elastic constants, and phase transition pressures are calculated and compared with the experimental data available and other theoretical results. According to linear-response approach, the thermodynamic properties such as the free energy, enthalpy, entropy, and heat capacity are also obtained successfully from the phonon density of state.
\end{abstract}

PACS: 71.15.Mb, 65.40.-b

\section{Introduction}

As a group IIB-VIA semiconductor, cadmium sulfide has gained wide recognition because of its outstanding optical-electronic properties [1-7] and polymorphic structural transformations [8-16]. It has a direct band gap of $2.4 \mathrm{eV}$ that lends itself to many applications in light detectors, forming quantum dots, and passivating the surfaces of other materials [1]. Owing to the thermal stability and the colour in yellow, it can form pigments in colors ranging from deep red to yellow with the addition of CdTe et al. [2]. At ambient conditions, CdS exists as hexagonal greenockite (wurtzite structure (WZ)) or cubic hawleyite (zincblende structure (ZB)). In this paper, we investigate the structures of $\mathrm{WZ}$ with space group $P 63 \mathrm{mc}$ ZB with space group $F-43 m$ and rocksalt structure (RS) with space group $F m-3 m$.

The electronic properties of CdS have been of considerable investigation by both theories $[3,4,7]$ and experiments $[5,6]$. Zakharov et al. [3] calculated quasiparticle band structures of CdS using the GW approximation for self-energy operator and obtained satisfactory agreement in comparison with experiments. Using the first-principles orthogonalized linear-combination-of-atomic orbitals method, Xu and Ching [4] calculated the energy gap of $2.02 \mathrm{eV}$, which is in reasonable agreement with experimental values of $2.4 \mathrm{eV}$ [5] and $2.58 \mathrm{eV}$ [6]. Wei et al. studied the structure stability and carrier localization of CdS in both ZB- and WZ-structures by band-structure calculations [7]. They found that the band gap and the valence-band maximum of WZ-CdS are larger

* corresponding author; e-mail: $j \cdot j \cdot \tan €$ tom.com than those of ZB-CdS, which can affect significantly device transport properties in a mixed $\mathrm{ZB} / \mathrm{WZ}$ system.

During the past few decades, the investigations of phase transitions have attracted considerable attention by experiments [8-15]. The pioneering study of optical-absorption edge of CdS under pressure indicated that the phase transition (WZ-RS) occurs at about $2.7 \mathrm{GPa}[8]$. In a subsequent resistivity study under pressure, a large drop in electrical resistance of WZCdS at about $2.5 \mathrm{GPa}$ was observed by Samara and Drickamer [9]. Corll reported that the phase transition pressures are very approximately at $3.1 \mathrm{GPa}$ (ZB-RS), 2.5 GPa (WZ-RS), and 2.8 GPa (WZ-ZB mixes - RS), respectively [10]. Using the resonant Raman scattering tuned by pressure, Zhao et al. and Venkateswaran et al. reported that the phase transition of $\mathrm{CdS}$ from a direct-gap WZ to an indirect-gap RS- structure at about 2.7 GPa [11] and 2.5 GPa [12]. Knudson et al. investigated the WZ-RS phase transition of CdS using picosecond time-resolved electronic spectroscopy in plate impact shock wave experiments [13]. They obtained the phase transition (WZ-RS) pressure of $3.1 \mathrm{GPa}$ by the Hartree-Fock linear-combination-of-atomic-orbitals self-consistent-field calculation and identified a face-centered tetragonal structure as the most plausible metastable structure. In addition, due to the interesting and size-dependent properties of nanocrystals of CdS semiconductor, the phase transition pressures of size-dependent nanocrystals of CdS were also studied. Makino et al. [14] measured the structural phase transition (WZ-RS) pressures of CdS microcrystals in various sizes and obtained the transition pressures between 6 and $7 \mathrm{GPa}$, which is larger than those of bulk crystals. By measuring the op- 
tical absorption of ZB-CdS microcrystals in $4 \mathrm{~nm}$ diameter, Haase and Alivisatos [15] reported that the phase transition pressure of $8 \mathrm{GPa}$, which outclasses the bulk CdS phase transition pressure of $3.1 \mathrm{GPa}$. The investigation on stability of different size nanocrystal CdS are expected to obtain the surface energy and explain the effects of strain and dislocation at the surface.

As described above, although the phase transitions of CdS have been extensively studied by experiments with different methods, the theoretical investigation $[13,16]$ on phase transitions of WZ- and ZB-CdS are relatively lacking. Thus, our work is respected to give a comparative and complementary theoretical support for experiments. A systematical investigation of high-pressure and high-temperature behaviors for CdS are given, which include the phase transition pressure, elasticity, aggregate elastic modulus, free energy, enthalpy, entropy, and heat capacity. Our calculations agree well with experimental results and provide powerful guidelines for further experimental investigation. This paper is organized as follows: in Sect. 2, we make a brief review of the theoretical methods. Subsequently, the results and some discussion are presented in Sect. 3. Finally, the conclusions are summarized in Sect. 4.

\section{Method of calculations}

\subsection{Total energy electronic structure calculations}

To perform density functional theory calculations, we employ the Vanderbilt-type ultrasoft pseudopotentials [17] for the interactions of the electrons and the ion cores, together with the local density approximation (LDA) for exchange and correlation in the scheme of Ceperley-Alder parameterized by Perdew and Zunger (CA-PZ) [18, 19]. The initial structures used in the condensed phase simulations are plotted in Fig. 1. The electronic wave functions are obtained using a density-mixing scheme [20] and the structures are relaxed using the Broyden-Fletcher-Goldfarb-Shannon (BFGS) method [21]. The electronic wave functions are expanded in a plane wave basis set with the energy cut-off of $400 \mathrm{eV}$. Hydrostatic pressure, coupled with the variable cell approach, is applied within the Parrinello-Rahman method to perform a full optimization of the cell structure [22]. For the Brillouin zone sampling, $8 \times 8 \times 8$ (for ZB- and RS-CdS) and $10 \times 10 \times 6$ (for WZ-CdS) Monkhorst-Pack meshes [23] and the self-consistent convergence $10^{-6} \mathrm{eV} /$ atom of the total energy are applied. Pseudoatom calculations are performed for Cd $4 d^{10} 5 s^{2}$ and $\mathrm{S} 3 s^{2} 3 p^{4}$. All the calculations are implemented through the CASTEP code [24, 25].

\subsection{Elastic properties and mechanical properties}

A brief description of computational methods of elastic constants is given as follows. The elastic stiffness tensor is related to the stress tensor and the strain tensor by Hooke's law. The elastic stiffness tensor $c_{i j k l}$ can be expressed as [26-29]:

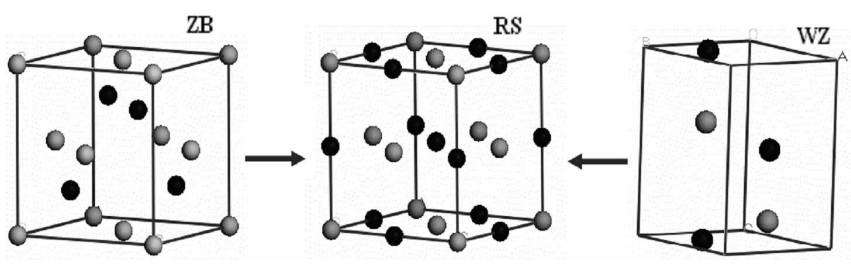

Fig. 1. Unit cell of CdS crystal. Gray and black spheres stand for Cd and S atoms, respectively.

$$
\sigma_{i j}=\sum_{k l}^{1,3} c_{i j k l} \varepsilon_{k l},
$$

where $\varepsilon_{k l}$ is the strain, $c_{i j k l}$ is the elastic constant (matrix) and $\sigma_{i j}$ is the stress. Generally speaking, the elastic stiffness tensor has 21 non-zero independent components. However, this number is greatly reduced when taking the symmetry of the crystal into account. As is known, there are three independent components of the elastic stiffness tensor for cubic crystal, i.e. $C_{11}, C_{12}, C_{44}$, and five independent components for hexagonal crystal, i.e. $C_{11}, C_{12}$, $C_{13}, C_{33}$, and $C_{44}$.

There are two approximation methods to calculate the polycrystalline modulus, namely the Voigt method [30] and the Reuss method [31]. The subscript $\mathrm{V}$ denotes the Voigt bound and $\mathrm{R}$ denotes the Reuss bound. For cubic phase CdS, the theoretical bulk modulus $B$ can be determined from elastic constants

$$
B_{\mathrm{V}}=B_{\mathrm{R}}=\left(C_{11}+2 C_{12}\right) / 3 \text {. }
$$

The shear modulus $G$ has the following form:

$$
\begin{aligned}
& G_{\mathrm{V}}=\left(C_{11}-C_{12}+3 C_{44}\right) / 5, \\
& G_{\mathrm{R}}=5\left(C_{11}-C_{12}\right) C_{44} /\left[4 C_{44}+3\left(C_{11}-C_{12}\right)\right] .
\end{aligned}
$$

For hexagonal phase CdS, the bulk modulus $B_{\mathrm{V}}$ and $B_{\mathrm{R}}$ are given by

$$
\begin{aligned}
& B_{\mathrm{V}}=(1 / 9)\left[2\left(C_{11}+C_{12}\right)+4\left(C_{13}\right)+C_{33}\right], \\
& B_{\mathrm{R}}=C^{2} / M .
\end{aligned}
$$

The shear modulus $G$ has the following expression:

$$
\begin{aligned}
& G_{\mathrm{V}}=(1 / 30)\left(M+12 C_{44}+12 C_{66}\right), \\
& G_{\mathrm{R}}=(5 / 2)\left[C^{2} C_{44} C_{66}\right] \\
& \quad /\left[3 B_{\mathrm{V}} C_{44} C_{66}+C^{2}\left(C_{44}+C_{66}\right)\right],
\end{aligned}
$$

where

$$
\begin{aligned}
& M=C_{11}+C_{12}+2 C_{33}-4 C_{13}, \\
& C^{2}=\left(C_{11}+C_{12}\right) C_{33}-2 C_{13}^{2} .
\end{aligned}
$$

The arithmetic average of the Voigt and the Reuss bounds is called the Voigt-Reuss-Hill (VRH) average and commonly used to estimate the elastic moduli of polycrystals. In the terms of the Voigt-Reuss-Hill approximations [32]:

$$
B_{\mathrm{V}}+B_{\mathrm{R}}=2 B_{\mathrm{H}}, G_{\mathrm{V}}+G_{\mathrm{R}}=2 G_{\mathrm{H}} .
$$

Young's modulus $E$ can be calculated by

$$
E=9 B G /(3 B+G) \text {. }
$$




\section{Results and discussion}

\subsection{Phase transition}

In order to investigate phase transitions, we analyze the enthalpy variation of CdS with pressure. Enthalpy $H$ is an important and valuable parameter for the study of phase stability. The phase transition pressure can be obtained by the enthalpy criterion, $H_{\text {phase } 1}\left(p_{\mathrm{t}}\right)=$ $H_{\text {phase } 2}\left(p_{\mathrm{t}}\right)$. Enthalpy $H$ takes the form of

$$
H=E+P V \text {, }
$$

where $E$ is the internal energy calculated by density functional theory, the pressure $P$ and volume $V$ are linked by the fourth-order Birch-Murnaghan equation of states.

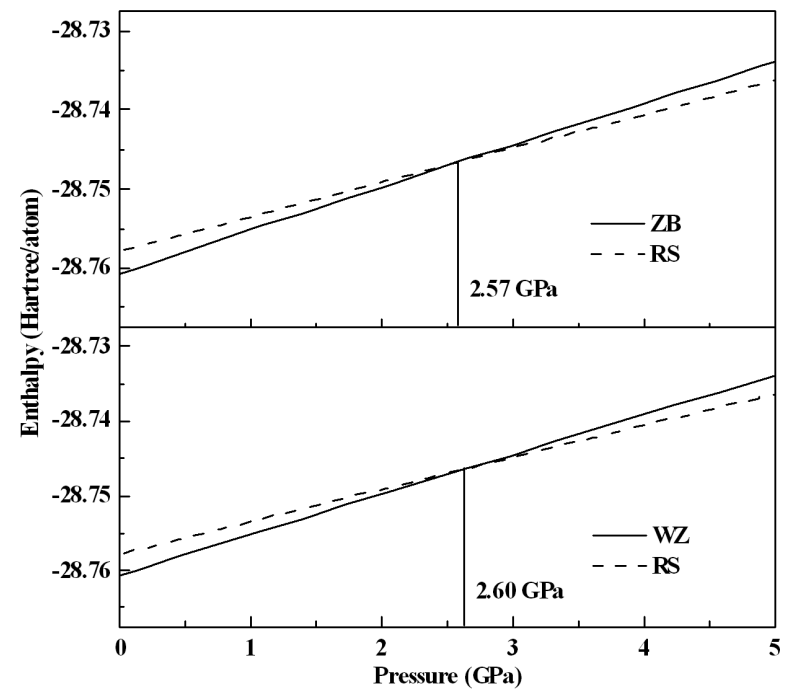

Fig. 2. The calculated enthalpy $(H)$ of ZB-, RS-, and WZ-CdS versus pressure.

By fitting the $E-V$ data to the Birch-Murnaghan equation of state (EOS) [33]

$$
\begin{aligned}
p & =3 B_{0} f_{E}\left(1+f_{E}\right)^{5 / 2}\left[1+\frac{3}{2}\left(B^{\prime}-4\right) f_{E}\right. \\
& \left.+\frac{3}{2}\left(B_{0} B^{\prime \prime}+\left(B^{\prime}-4\right)\left(B^{\prime}-3\right)+\frac{35}{9}\right) f_{E}^{2}\right],
\end{aligned}
$$

where $f_{E}$ is written as

$$
f_{E}=\left[\left(V_{0} / V\right)^{2 / 3}-1\right] / 2,
$$

where $E$ is the internal energy calculated by density functional theory, the pressure $P$ and volume $V$ are linked by the fourth-order Birch-Murnaghan equation of states. We calculate the variations of the enthalpy of $\mathrm{CdS}$ versus the pressure, which are shown in Fig. 2. The intersection of the two enthalpy curves implies that the phase transitions occur at $2.57 \mathrm{GPa}(\mathrm{ZB}-\mathrm{RS})$ and $2.60 \mathrm{GPa}$ (WZ-RS), respectively. We list the available experimental data and other theoretical results for comparison in Table I. It can be found from Table I, our computational values are in good agreement with experimental data [10$12,15,34]$ and previous theoretical investigation $[13,16]$.

\subsection{Crystallographic structure of $C d S$ at ground state}

The ground state crystallographic structure is important and fundamental for investigating the other properties. Ascertaining the equilibrium parameters of CdS at ambient pressure is important not only for the knowledge of the molecular crystal structure at ambient condition, but also to benchmark the performance of the theoretical methods for its ability to describe interatomic binding forces in solids. A series of lattice constants $a$ are set to obtain the total energy $E$ and the corresponding primitive cell volume $V$, and then the obtained $E-V$ data are fitted to the Birch-Murnaghan equation of state [33]. The obtained equilibrium lattice parameters $a$ and $c$, bulk modulus $B_{0}$ and its pressure derivation $B_{0}^{\prime}$ at $P=0$ and $T=0$ are listed in Table II, together with the available experimental [35-37] and other theoretical $[13,16,38]$ data. Compared with the experimental data, our LDA calculations underestimate lattice constants $a$ by $-1.21 \%$ (WZ), $-0.72 \%(\mathrm{ZB}),-1.51 \%$ (RS), respectively. Generally speaking, our computational results are consistent with the experimental data and other theoretical results.

\subsection{Elastic properties and mechanical properties under pressure}

The elastic constants of solids provide a link between the mechanical and dynamical behaviors of crystals, and give important information concerning the nature of the forces operating in solids. Our calculated elastic constants of CdS at $P=0$ and $T=0$ are summarized in Table III, together with the available experimental and other theoretical data. Our calculated elastic constants of WZ CdS approach to the experimental data [39] and are generally better than those given by Wright and Gale [38].

The good agreement between ours and experiments implies the validity of aggregate elastic modulus under high pressure, which is shown in Fig. 3. It is obvious that the elastic modulus $C_{11}, C_{12}, C_{13}$, and $C_{33}$ increase linearly with pressure increasing, but the elastic constant $C_{44}$ changes slightly. These properties can give some information for the applications in extreme environments.

In Fig. 4, we plot the variation of bulk modulus $B$, shear modulus $G$, and Young's modulus $E$ as a function of the pressure. The bulk modulus of ZB-, WZ-, and RS-CdS increases linearly with pressure increasing. The shear modulus and Young's modulus of ZB- and WZ-CdS decrease with pressure increasing, while those of RS-CdS increase with pressure increasing. They exhibit abrupt changes at $1 \mathrm{GPa}$ for ZB-CdS and $3 \mathrm{GPa}$ for WZ-CdS. The abrupt changes originate from the sensitivity of $C_{44}$ with the variation of pressure. 
The pressure (in GPa) of phase transiton of CdS.

TABLE I

\begin{tabular}{c|c|c|c}
\hline \hline Phase transition & Pt & Method & References \\
\hline ZB-RS & 2.57 & LDA/USP & present work \\
& 2.1 & FP/LMTO & Benkhettou et al. Ref. [16] \\
& 2.7 & Raman scattering & Haase et al. Ref. [15] \\
& 3.1 & X-ray diffraction & Corll Ref. [10] \\
WZ-RS & 2.60 & LDA/USP & present work \\
& 2.3 & FP/LMTO & Benkhettou et al. Ref. [16] \\
& 3.1 & HF/LYP & Knudson et al. Ref. [13] \\
& 2.5 & X-ray diffraction & Owen et al. Ref. [34] \\
& 2.7 & Raman scattering & Zhao et al. Ref. [11] \\
& 2.5 & Raman scattering & Venkateswaran et al. Ref. [12] \\
& 2.5 & X-ray diffraction & Corll Ref. [10]
\end{tabular}

TABLE II

The calculated lattice constants $(\AA)$, the bulk modulus $B_{0}$ (in GPa) and its first pressure derivative $B_{0}^{\prime}$ of $\mathrm{CdS}$ at $0 \mathrm{GPa}$ and $0 \mathrm{~K}$.

\begin{tabular}{c|c|c|c|c|c}
\hline \hline Structure & References & $a$ & $c$ & $B_{0}$ & $B_{0}^{\prime}$ \\
\hline WZ & present work & 4.086 & 6.667 & 68.9 & 4.7 \\
& Ref. [13] & 4.18 & 6.76 & 79.48 & 2.941 \\
& Ref. [38] & 4.19 & 6.66 & 66.4 & \\
& Expt. Ref. [35] & 4.136 & 6.714 & 62.0 & \\
ZB & present work & 5.776 & & 68.1 & 4.7 \\
& Ref. [16] & 5.8081 & & 69.44 & 4.5656 \\
& Ref. [38] & 5.87 & & 65.5 & \\
& Expt. Ref. [35] & 5.818 & & $64.4[36]$ & \\
& present work & 5.358 & & 92.5 & 4.8 \\
& Ref. [16] & 5.3527 & & 97.28 & 4.5101 \\
& Ref. [13] & 5.45 & & 94.74 & 3.802 \\
& Expt. Ref. [37] & 5.44 & & 86.7 & 4.36
\end{tabular}

The calculated elastic constants (in GPa) of CdS at $0 \mathrm{GPa}$ and $0 \mathrm{~K}$.

TABLE III

\begin{tabular}{c|c|c|c|c|c|c}
\hline \hline Structure & References & $C_{11}$ & $C_{12}$ & $C_{13}$ & $C_{33}$ & $C_{44}$ \\
\hline WZ & present work & 93.93 & 57.64 & 50.19 & 105.24 & 15.87 \\
& Ref. [38] & 102.8 & 45.4 & 47.5 & 113.3 & 32.4 \\
& Expt. Ref. [39] & 84.32 & 52.12 & 46.38 & 93.97 & 14.89 \\
ZB & present work & 81.79 & 61.06 & & & 31.0 \\
& Ref. [38] & 89.38 & 53.52 & & & 39.11 \\
RS & present work & 157.82 & 59.66 & & & 35.33
\end{tabular}

\subsection{Thermodynamic properties}

Phonons play a major role in many physical properties of solids, such as the thermal property, electrical conductivity, superconducting temperature and the Debye temperature. The vibration frequency is a function of the wave vector $q$, which can be written as: $\omega(q)=\omega_{j}(q)$. Due to the translational symmetry $\omega_{j}(q+G)=\omega_{j}(q)$, we can only calculate the phonon frequencies in the first Brillouin zone.
Once the phonon spectra are obtained, we can calculate the Helmholtz free energy $F$, entropy $S$ and lattice capacity $C_{\mathrm{V}}$ as a function of temperature as follows:

$$
E(T)=E_{\mathrm{tot}}+E_{\mathrm{zp}}+\int \hbar \omega /\left[\exp \left(\frac{\hbar \omega}{k T}\right)-1\right] g(\omega) \mathrm{d} \omega
$$

with $E_{\text {tot }}$ and $E_{\mathrm{zp}}$ representing initial energy and zero point vibrational energy, $k$ and $h$ denoting Boltzmann's and Planck's constant. $g(\omega)$ is the phonon density of 

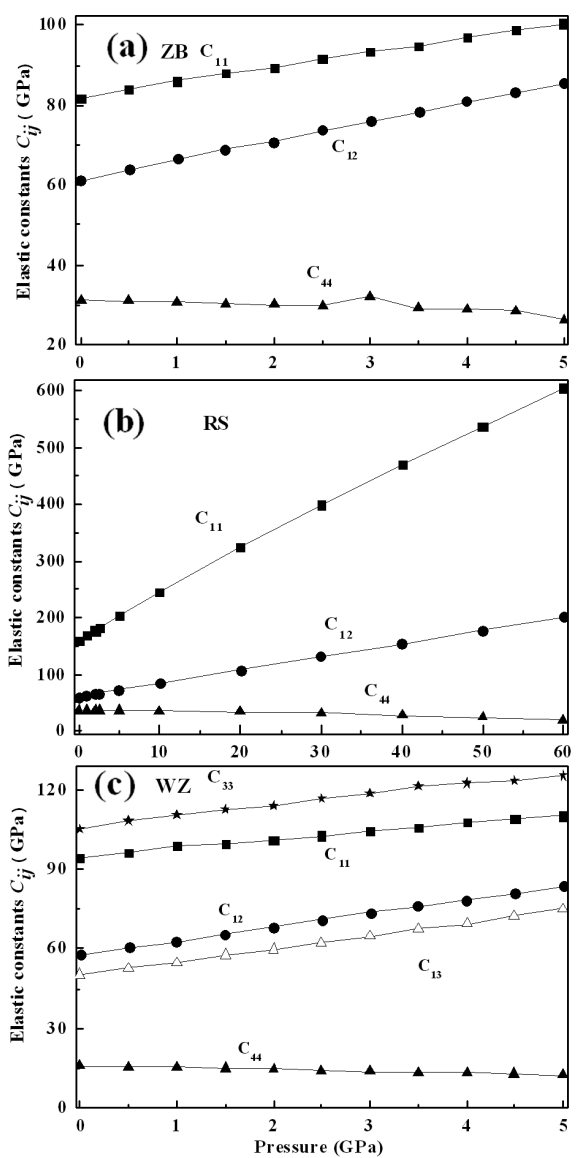

Fig. 3. The calculated pressure dependence of elastic constants $C_{11}$ (solid squares), $C_{12}$ (solid circles), $C_{44}$ (solid triangles), $C_{33}$ (solid stars), $C_{13}$ (triangles) at $0 \mathrm{~K}$ of CdS.

states with $\int_{0}^{\omega} g(\omega) \mathrm{d} \omega=1 . E_{\text {zp }}$ can be expressed by

$$
E_{\text {zp }}=\frac{1}{2} \int \hbar \omega g(\omega) \mathrm{d} \omega .
$$

The vibrational contribution to the free energy $F$ is given by

$$
\begin{aligned}
& F(T)=E_{\text {tot }}+E_{\text {zp }}+k T \int\left[1-\exp \left(-\frac{\hbar \omega}{k T}\right)\right] \\
& \quad \times \operatorname{lmg}(\omega) \mathrm{d} \omega .
\end{aligned}
$$

The vibrational contribution to the entropy $S$ can be written in the form of

$$
\begin{aligned}
& S(T)=k\left\{\int \frac{\hbar \omega}{k T} /\left[\exp \left(\frac{\hbar \omega}{k T}\right)-1\right] g(\omega) \mathrm{d} \omega\right. \\
& \left.-\int\left[1-\exp \left(-\frac{\hbar \omega}{k T}\right)\right] g(\omega) \mathrm{d} \omega\right\} .
\end{aligned}
$$

The vibrational contribution to the heat capacity $C_{\mathrm{V}}$ is deduced by

$$
\begin{aligned}
& C_{\mathrm{V}}(T)=k \int\left(\frac{\hbar \omega}{k T}\right)^{2} \exp \left(\frac{\hbar \omega}{k T}\right) /\left[\exp \left(\frac{\hbar \omega}{k T}\right)-1\right]^{2} \\
& \quad \times g(\omega) \mathrm{d} \omega .
\end{aligned}
$$

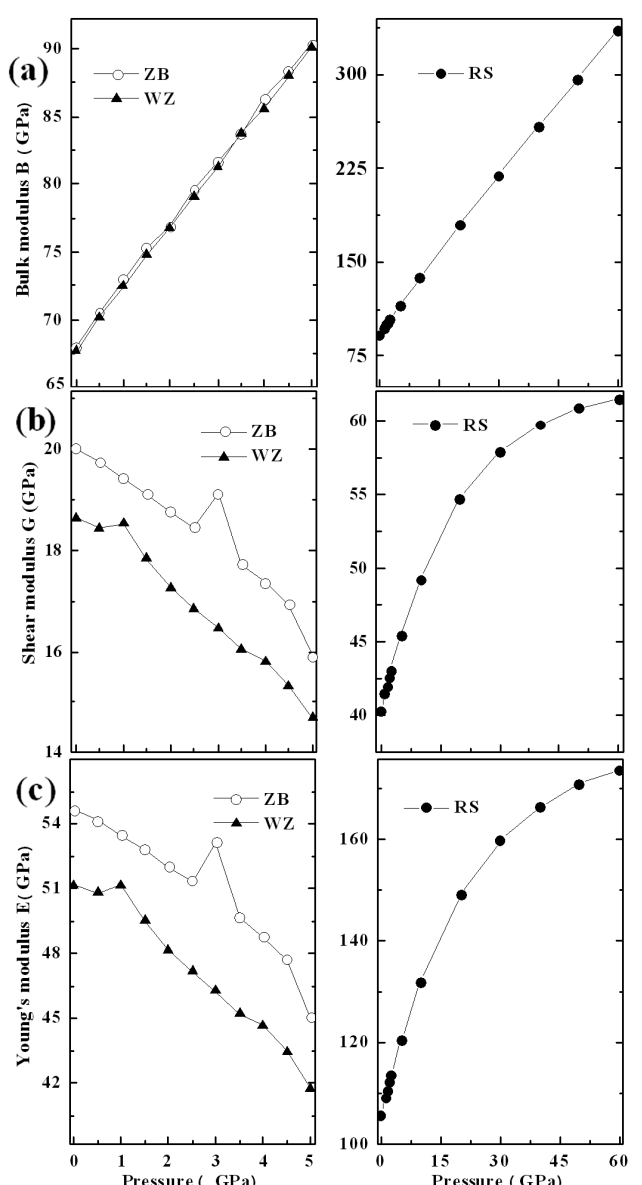

Fig. 4. The calculated bulk modulus $B$, shear modulus $G$, and Young's modulus $E$ of ZB- (circles), RS- (solid circles), and WZ- (solid triangles) CdS as a function of pressure.

We plotted the thermodynamic functional in Fig. 5 . All the investigated thermodynamic data of ZB- and WZ-CdS are much similar to each other. The predicted high-temperature behaviors can give some information for applications of the materials at different temperatures.

\section{Conclusions}

In summary, we have presented structural phase transitions equations of state and elasticity for ZB, WZ, and RS CdS by ab initio plane-wave pseudopotential calculations. The phase transition pressures are $2.57 \mathrm{GPa}$ (ZB-RS), and 2.60 GPa (WZ-RS). The effects of the pressure on the elastic constants and mechanical properties have also been calculated. These results can give a better understanding of the high-pressure behavior of CdS. All the investigated thermodynamic data for ZBand WZ-CdS are much similar to each other. These results can provide powerful guidelines for a further understanding of vibrational contribution to the temperature dependence of the thermodynamic potentials. 

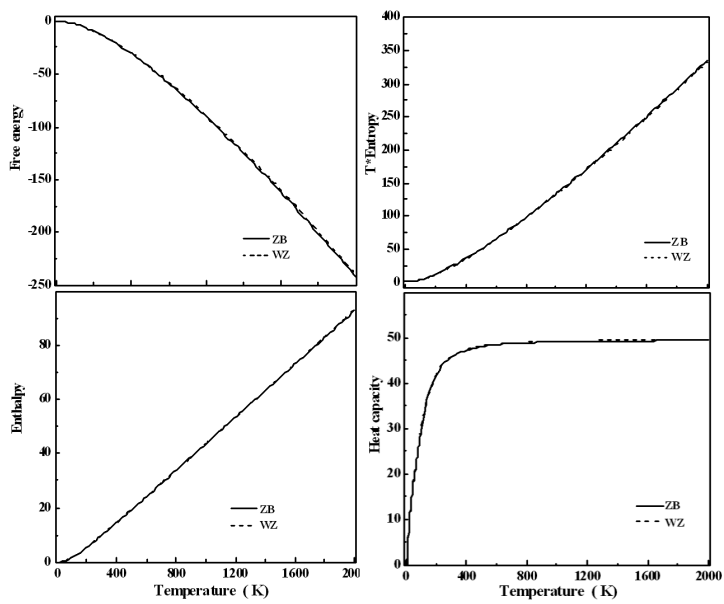

Fig. 5. The calculated temperature dependence of the vibrational contribution to the free energy $F$ (KJ $\left.\mathrm{mol}^{-1}\right)$, enthalpy $H\left(\mathrm{KJ} \mathrm{mol}^{-1}\right)$, entropy $T S$ $\left(\mathrm{KJ} \mathrm{mol}{ }^{-1}\right)$, and heat capacity $C_{v}\left(\mathrm{~J} \mathrm{~mol}^{-1} \mathrm{~K}^{-1}\right)$ of the ZB- and WZ-CdS.

\section{Acknowledgments}

The authors would acknowledge the Specialized Research Fund for the Doctoral Program of Higher Education under grant No. 20090181110080.

\section{References}

[1] M. Fernee, A. Watt, J. Warner, S. Cooper, N. Heckenberg, H. Rubinsztein-Dunlop, Nanotechnology 14, 991 (2003).

[2] N.N. Greenwood, A. Earnshaw, Chemistry of the Elements, 2nd ed., Butterworth-Heinemann, Oxford 1997.

[3] O. Zakharov, A. Rubio, X. Blasé, M.L. Cohen, S.G. Louie, Phys. Rev. B 50, 10780 (1994).

[4] Y.N. Xu, W.Y. Ching, Phys. Rev. B 48, 4335 (1993).

[5] M.J. Weber, Handbook of Laser Science and Technology, Vol. III CRC, Cleveland 1986.

[6] L. Ley, R.A. Pollak, F.R. Mcfeely, S.P. Kowalczyk, D.A. Shirley, Phys. Rev. B 9, 600 (1974). As cited therein.

[7] S. Wei, S.B. Zhang, Phys. Rev. B 62, 6944 (2000).

[8] A.L. Edwards, H.G. Drickamer, Phys. Rev. 122, 1149 (1961).

[9] G.A. Samara, H.G. Drickamer, J. Phys. Chem. Solids 23, 457 (1962).

[10] J.A. Corll, J. Appl. Phys. 35, 3032 (1964).

[11] X.S. Zhao, J. Schroeder, T.G. Bilodeau, L.G. Hwa, Phys. Rev. B 40, 1257 (1989).

[12] U. Venkateswaran, M. Chandrasekhar, Phys. Rev. B 31, 1219 (1985).

[13] M.D. Knudson, Y.M. Gupta, A.B. Kunz, Phys. Rev. B 59, 11704 (1999).
[14] T. Makino, K. Matsuishi, S. Onari, T. Arai, J. Phys., Condens. Matter 10, 10919 (1998).

[15] M. Haase, A.P. Alivisatos, J. Phys. Chem. 96, 6756 (1992).

[16] N. Benkhettou, D. Rached, B. Soudini, M. Driz, Phys. Status Solidi B 241, 101 (2004).

[17] D. Vanderbilt, Phys. Rev. B 41, 7892 (1990).

[18] S.H. Vosko, L. Wilk, M. Nusair, Can. J. Phys. 58, 2100 (1980).

[19] D.M. Ceperley, B.J. Alder, Phys. Rev. Lett. 45, 566 (1980).

[20] G. Kresse, J. Furthmuller, Phys. Rev. B 54, 11169 (1996).

[21] T.H. Fischer, J. Almlof, J. Phys. Chem. 96, 9768 (1992).

[22] H.J. Monkhorst, J.D. Pack, Phys. Rev. B 13, 5188 (1976).

[23] M. Parrinello, A. Rahman, Phys. Rev. Lett. 45, 1196 (1980).

[24] M.C. Payne, M.P. Teter, D.C. Allen, T.A. Arias, J.D. Joannopoulos, Rev. Mod. Phys. 64, 1045 (1992).

[25] V. Milman, B. Winkler, J.A. White, C.J. Packard, M.C. Payne, E.V. Akhmatskaya, R.H. Nobes, Int. J. Quantum Chem. 77, 895 (2000).

[26] J. Wang, S. Yip, S.R. Phillpot, D. Wolf, Phys. Rev. Lett. 71, 4182 (1993).

[27] D.C. Wallace, Thermodynamics of Crystals, Wiley, New York 1972.

[28] B.B. Karki, G.J. Ackland, J. Crain, J. Phys., Condens. Matter 9, 8579 (1997).

[29] T.H.K. Barron, M.L. Klein, Proc. Phys. Soc. 85, 523 (1965).

[30] W. Voigt, Lehrbuch der Kristallphysik, B.G. Teubner, Leipzig 1910 (in German).

[31] A.Z. Reuss, Z. Angew. Math. Mech. 9, 55 (1929).

[32] R. Hill, Proc. Phys. Soc. Lond. 65, 350 (1952).

[33] F. Birch, Phys. Rev. 71, 809 (1947).

[34] N.B. Owen, P.L. Smith, J.E. Martin, A.J. Wright, J. Phys. Chem. Solids 24, 1519 (1963).

[35] Numerical Data and Functional Relationships in Science and Technology, Eds. O. Madelung, M. Schölz, H. Weiss, Landolt-Börnstein, Vol. 17, Springer, Berlin 1982.

[36] F. Bechstedt, W.A. Harrison, Phys. Rev. B 39, 5041 (1984).

[37] T. Suzuki, T. Yagi, S. Akimoto, T. Kawamura, S. Toyoda, S. Endo, J. Appl. Phys. 54, 748 (1983).

[38] K. Wright, J.D. Gale, Phys. Rev. B 70, 035211 (2004).

[39] D.I. Bolef, N.T. Melamed, M. Menes, J. Phys. Chem. Solids 17, 143 (1960). 